\title{
A LOCALIZATION ALGORITHM FOR STRONG SOURCES OF ORBIT MOTION IN THE APS STORAGE RING*
}

\author{
C. Schwartz ${ }^{\dagger}$, J. Carwardine, and T. Fors \\ Advanced Photon Source, Argonne National Laboratory, Argonne, IL
}

\begin{abstract}
This paper describes a semi-automatic algorithm that locates spatially discrete strong sources of storage-ring noise that cause beam motion, e.g., noisy power supplies. The algorithm parasitically uses the APS real-time orbit feedback system (RTFS) to obtain orbit data on line, and a response matrix model to compute the confidences for source candidates. It also computes the estimated spectral density of the source. Further rules-of-thumb for source identification and empirical data are also presented.
\end{abstract}

\section{INTRODUCTION}

There is an ongoing effort at the APS to improve the position stability of $\mathrm{x}$-ray beams delivered to the users. An important component of that effort is to minimize the storage-ring beam motion [1], which itself is caused by countless parallel unknown noise sources. Some sources affect all of the ring at once (e.g., seismic noise), while others are spatially discrete in nature, such as any of 1,400 magnet power supplies. The new APS glitch detection system [2] is one means to find faulty power supplies. This paper presents a complementary algorithm, one that localizes sources using large-bandwidth transverse-motion data from the beam itself and response matrix models.

\section{THEORETICAL CONSIDERATIONS}

\subsection{Source Characteristics}

Possible motion sources include over 300 correctors, which by design induce transverse motion. Multipole magnets (i.e., quadrupoles and sextupoles) also induce transverse motion, largely horizontal motion, as a consequence of the vacuum chamber asymmetry [3]. Correctors have a near-unity small-signal gain factor, i.e., a 1-A current step change-from-nominal induces beam motion on the order of $1 \mathrm{~mm}$, while the conversion factor for quadrupoles and sextupoles is an order of magnitude smaller and is frequency dependent. Thus, while multipole magnets are possible sources, they must have noise levels an order of magnitude larger than that of correctors for the same level of detectability.

A further peculiarity of the APS vacuum chamber is that its properties distinguish the response speeds of certain correctors and lattice elements. There are aluminum

\footnotetext{
* Work supported by U.S. Department of Energy, Office of Basic Energy Sciences, under Contract No. W-31-109-ENG-38.

${ }_{\dagger}^{\dagger}$ csch@aps.anl.gov
}

and Inconel sections: the former have a much slower eddycurrent-penetration response time. All of the lattice elements except the "fast" ( 38 per plane) RTFS correctors straddle aluminum sections and have a response roll-off at around $5 \mathrm{~Hz}$, while the fast correctors' response is an order of magnitude quicker. The upshot of these data is that vertical plane motion induced by a large-amplitude lattice-element source is caused by correctors. Likewise, horizontal-plane, high-frequency or broadband $(>10 \mathrm{~Hz})$ discrete-lattice-element motion is likely caused by a horizontal corrector source. Low-frequency horizontal plane motion can be from a (high-noise-level) multipole magnet. Thus, our ability to localize sources is aided greatly by time/frequency data, in addition to static response-matrix type data.

\subsection{Response Matrix Inversion}

In each plane, the $M \times N$ response matrix maps all $N$ sources to all $M \sim 300$ beam position monitors (BPMs). Thus, in theory, one can estimate the ( $N$-tuple) noise time series that generated the BPM data by pseudo-inverting the full matrix and multiplying the result by a matrix-array of BPM data. However, as discussed below, our algorithm implementation is limited by signal dimension constraints: we can currently obtain only $m=38 \ll M$ channels of large-bandwidth BPM data. We therefore use a $(m \times n)$ pruned version of the response matrix, denoted $\mathbf{P}$. A sufficient condition for full rank (in the case of the response matrix) is $n \leq m$, which is assumed henceforth.

However, real-world robust computations require that the columns of $\mathbf{P}$ be numerically linearly independent. The degree of linear independence is quantified by the (2-norm) condition number $\kappa(\mathbf{P})$; formally, the ratio of maximum and minimum singular values of $\mathbf{P}$ [4]. We have found that $\kappa(\mathbf{P})$ on the order of $10^{5}$ or higher leads to inaccurate results; a desired value is on the order of $10^{3}$.

A first step in improving $\mathbf{P}$ 's conditioning is to remove columns that correspond to correctors on the same girder (which are nearly linearly dependent). A girder corresponds to $1 / 200$ th of the ring circumference, or $1 / 5$ th of a lattice period. Thus, instead of attempting source identification, we attempt localization to the girder level; a suspect girder implies that all of the correctors on the girder are suspect (between 1 and 2). Although removing girdersharing correctors reduces $\kappa$ by orders of magnitude, it is still too large. Fig. 1 is useful for visualizing this problem and suggests a means of sidestepping it. Shown in (top) are two correctors from adjacent girders across all BPMs, 

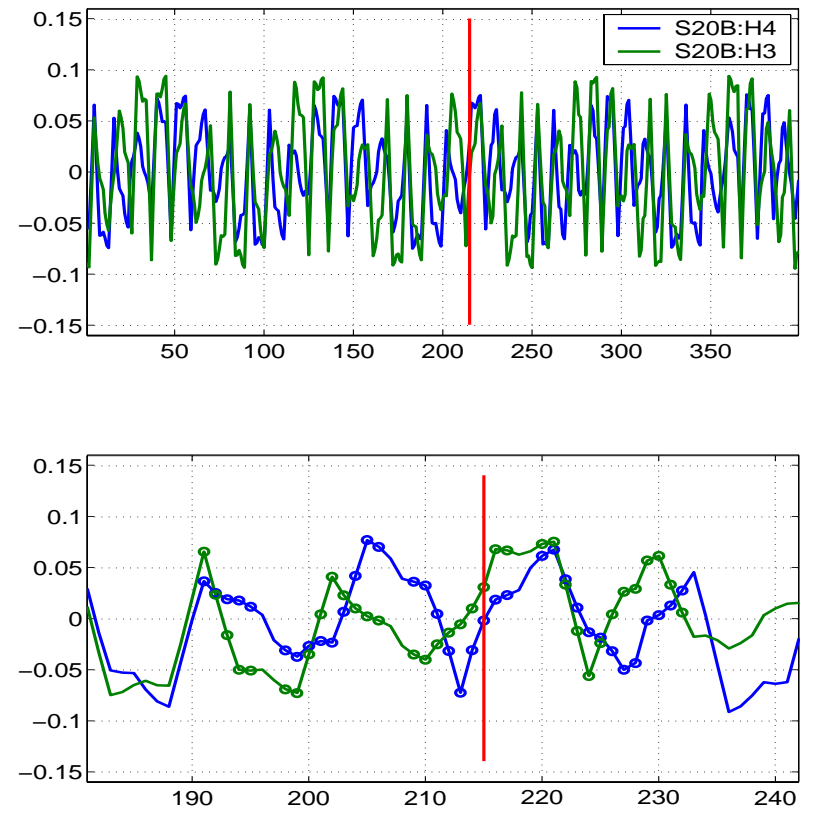

Figure 1: The response (in $\mathrm{mm}$ ) of S20B:H4 and its downstream neighbor S20B:H3, as seen on all of the BPMs (top), and on 38 nearby BPMs, marked with "o" (bottom). The red vertical line indicates the approximate spatial location of the two correctors.

while (bottom) is a zoom on the local properties of the correctors' response. Allowing for only $m=38$ spatial samples of each corrector response, it is clear that a localized reading resolves the two responses better than an evenly (spatially) sampled one. The inner product of the former is five-fold smaller ("five times more linearly independent") than the latter (0.16 to 0.08$)$. Using local BPMs also reduces the algorithm's dependence on particular BPMs, thus decreasing sensitivity to BPM noise.

To summarize, we can improve the conditioning of the response matrix by (1) neglecting girder-sharing correctors, and (2) using BPM orbit data near the source. However, we can attain a truly well-conditioned matrix $(\kappa<1,000)$ by (3) limiting the localization search to sources within a three-sector arc (or $7.5 \%$ of the ring circumference). Assuming the noise source can first be localized to within the desired range, it can subsequently be localized further to the girder level using an appropriate $\mathbf{P}$. Below we show that this assumption is legitimate.

Although the discussion in this subsection addressed correctors and not multipole magnets, in our experiments we have found that under the above three conditions, multipole sources whose waveforms are time-varying can be treated as additional girder-sharing correctors.

Finally, it should be pointed out that the above analysis ignores the twin effects of global orbit feedback. The first effect is to effectively filter the source's waveform. The second effect is to spatially correct the source, effectively altering its resemblance to columns of the response matrix. Both effects make source localization more difficult, although the second effect is largely mitigated by using BPM signals near the source, as the feedback is more effective globally than locally. In principle, it is possible to "undo" the twin effects of feedback by matrix filtering the BPM data (with the feedback system(s)'s inverse transfer function matrix [5]); however, to date, this has not brought much improvement in algorithm performance.

\section{IMPLEMENTATION AND EXPERIMENTAL RESULTS}

For the reasons outlined in the previous section, we have implemented a two-step source localization algorithm, as detailed below. The data-acquisition mechanism is via the 38-channel "DSPScope" facility of the APS RTFS, which allows for variable-bandwidth BPM and "corrector-error" data. The corrector-error vector is the instantaneous correction load (in A) for all of the 38 RTFS correctors; for each tick $k\left(T_{\mathrm{s}}=650 \mu \mathrm{s}\right)$, the corrector error is given by (see [3], [5]) $\mathbf{c}_{\mathrm{E}}[k]=\mathbf{R}_{\mathrm{rt}}^{+} \mathbf{b}_{\mathrm{rt}}[k]$, where $\mathbf{R}_{\mathrm{rt}}\left(\mathbf{b}_{\mathrm{rt}}\right)$ is the $160 \times 38$ RTFS response matrix $(160 \times 1$ vector of BPM readings). The ${ }^{+}$denotes the pseudo inverse operation [4]. Different frequency ranges are obtained by decimating the sampling rate (a built-in averaging filter mitigates aliasing).

Thus, the first step of our two-step algorithm is to use corrector error data to localize the source down to roughly the sector level (=1/40th of the ring). The second step utilizes BPM data and a sector-local response matrix $\mathbf{P}$ to further localize the source down to the girder level. We assume that the spectral properties of the source are unchanged in these two steps.

\subsection{Sector Localization}

Sector localization only roughly follows the corrector error. A particular source induces a relatively large corrector error in its own sector and \pm 1 adjacent sectors, i.e., 15 girders. However, we can further ignore some of the girders surrounding the sector that has the maximum corrector error.

Figure 2 shows some in-situ sector 20 (normalized) RMS RTFS corrector-error data for the horizontal plane; the results shown resemble those for all of the sectors in the ring. The theoretical data were generated by the matrix multiplication $\mathbf{R}_{\mathrm{rt}}^{+} \mathbf{P}$, where $\mathbf{P}$ now denotes the mapping of all sector-20 girder-unique correctors to the RTFS BPMs.

The figure bears out the fact that some correctors, e.g., the A:H1, map well to their own sector as corrector errors, however, others map to the downstream-adjacent corrector error, at maximum three girders away. In short, the maximum corrector-error RMS is not a reliable indicator of the sector location of the source, but it is only off by a maximum of three girders in the horizontal plane (a similar analysis shows that it is off by maximally one girder in the vertical plane). Thus, in the first step of the algorithm, the user records some corrector-error data and selects a noisy sector, with the understanding that the discrete noise source 

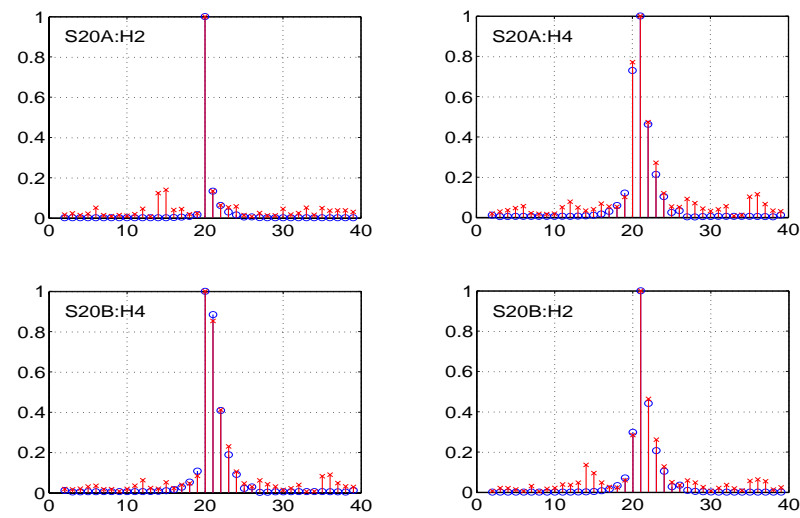

Figure 2: Sectors 2-39 empirical (x) and theoretical (o) normalized horizontal-plane corrector-error RMS caused by certain sources.

(if any) is in that sector \pm 3 or 1 adjacent girders for the horizontal and vertical planes, respectively.

\subsection{Girder Localization}

The second step of the algorithm localizes the source further using new BPM data. Specifically, 38 BPM data records are mapped to 11 possible horizontal (or 8 vertical) girders via the pseudo-inverse response matrix $\mathbf{P}^{+}$. The power of each estimated source channel gives a relative confidence of the source. The BPM data are further used to estimate the source spectral density, whose properties can be used to rule out multipole types (broadband and high-frequency noise [ $>10 \mathrm{~Hz}]$ is likely from a corrector and not from a multipole magnet).

The algorithm has been coded up in Tcl/Tk, and interfaces with the SDDS toolkit introduced in [6]. It also utilizes a stand-alone (compiled) Matlab-graphics module, which allows arbitrary zoom and rotate operations of a 3D mesh plot of corrector-error spectral densities, such as the one shown in [3]. This tool allows the user to visualize the location, frequency-dependence, and intensity of noise around the ring.

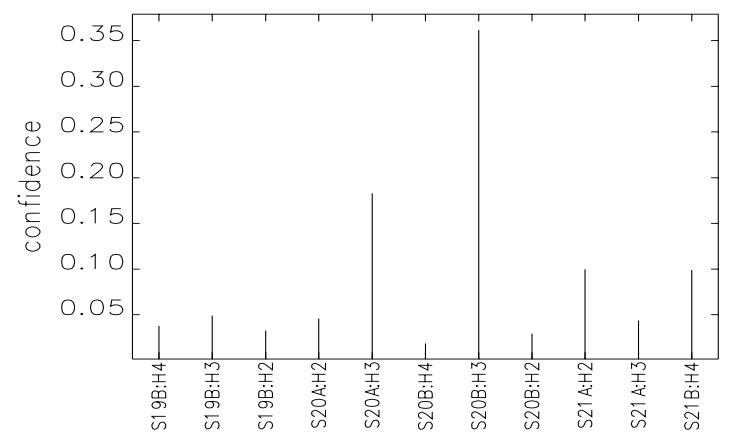

Figure 3: The algorithm's guess of the offending corrector or girder in terms of confidence.

\subsection{Some Empirical Results}

By pinging corrector S20B:H3 with a \pm 4 A triangle wave of period $10 \mathrm{~s}$, a corrector-error histogram similar to that of S20B:H4 shown in Fig. 2 was observed. The user thus assumes an error in sector 21 or thereabouts. After this user choice, the algorithm proceeds to take BPM data and then computes the likely girder source. The algorithm script then returns the two plots shown in Figs. 3 and 4, namely, the confidence in the estimation and a spectrum, scaled to reflect sine-wave amplitude. The algorithm's accuracy is $100 \%$ for closed-loop, in-situ tests of each corrector type (there are eight) when the source's amplitude is on the order of 1-4 A. Multipole magnets require higher current levels to achieve the same accuracy, as larger current levels are required to achieve a similar magnitude of beam motion.

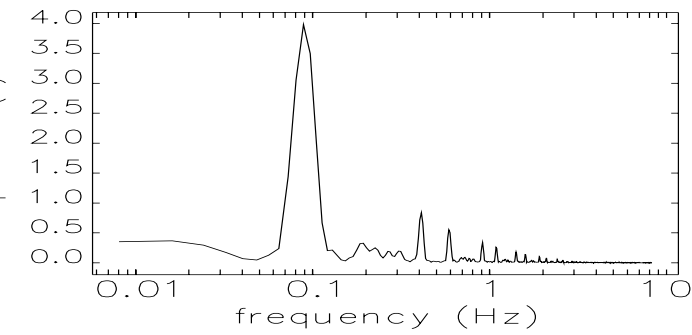

Figure 4: Estimated spectrum of the source computed from the BPM signals.

\section{ACKNOWLEDGMENTS}

The authors wish to acknowledge the work of R. Seglem, D. Donkers, and R. Vargas, of the APS Power Supply Group, who assisted in performing measurements on suspect power supplies. L. Emery also helped with technical discussions.

\section{REFERENCES}

[1] G. Decker and O. Singh, "Strategy for Achieving True rms Orbit Stabilization at the Advanced Photon Source," these proceedings.

[2] T. Fors, et al. "A Method of Measuring Noise and Detecting Glitches in Magnet Power Supplies at the APS Storage Ring," these proceedings.

[3] J.A. Carwardine and F.R. Lenkszus, "Real-Time Orbit Feedback at the APS," Proc. Eighth Beam Instrumentation Workshop (1998).

[4] G. H. Golub and C. F. Van Loan, Matrix Computations, Second Ed. (1989).

[5] C. Schwartz and L. Emery, "Compensating the Frequency Deadband of the APS Real-Time and DC Transverse Orbit Correction Systems," these proceedings.

[6] M. Borland, "A Self-Describing File Protocol for Simulation Integration and Shared Post-Processors," 1995 Particle Accelerator Conference (1996) p. 2184. 